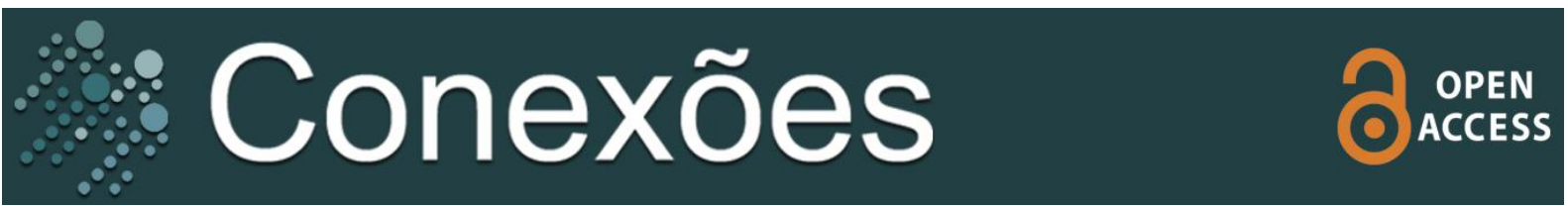

Artigo Original

\title{
Determinantes do desempenho esportivo na ginástica de trampolim
}

\author{
Determinants of Sport Performance in Trampoline Gymnastics
}

Determinantes del rendimiento deportivo en la gimnasia de trampolín

Ana Luíza Ferreira ${ }^{1}$
Paulo Carrara ${ }^{2}$

Kerly Priscila Jesus de Oliveira ${ }^{1}$

Newton Santos Vianna Júnior ${ }^{3}$

Francisco Zacaron Werneck ${ }^{1}$

\section{RESUMO}

Introdução: encontrar crianças com elevado potencial esportivo é uma importante etapa para o processo de formação esportiva, pois é o primeiro passo na descoberta de novos talentos visando o alto rendimento. O objetivo foi investigar os determinantes do desempenho na ginástica de trampolim e sua importância atribuída de acordo com os aparelhos da modalidade. Metodologia: participaram 40 experts (treinadores, gestores e árbitros) de ginástica de trampolim com experiência nacional e internacional. Os experts responderam sobre a importância atribuída aos fatores antropométricos, físico-motor, técnico, tático, psicológico e socioambiental. Avaliaram a importância dos fatores e indicadores de desempenho nos aparelhos trampolim acrobático, tumbling e duplo minitrampolim. Resultados e discussão: os determinantes do desempenho em ordem de importância decrescente foram: físico-motores, técnicos, psicológicos, táticos, antropométricos e socioambientais, com variação entre os aparelhos. Os indicadores de desempenho: massa muscular, velocidade, agilidade, flexibilidade, equilíbrio e força rápida de membros superiores apresentaram diferenças entre os aparelhos. Conclusão: revelouse a opinião dos experts sobre quais os fatores e indicadores de desempenho são os mais importantes na ginástica de trampolim com implicações na deteç̧ão e seleção de talentos.

Palavras-chave: Talento. Esportes Juvenis. Performance Esportiva.

${ }^{1}$ Universidade Federal de Ouro Preto, Escola de Educação Física, Laboratório de Estudos e Pesquisas do Exercício e Esporte, Ouro Preto - MG, Brasil.

2 Universidade de São Paulo, Escola de Artes, Ciências e Humanidades, São Paulo - SP, Brasil.

3 Federação Mineira de Ginástica. Belo Horizonte - MG, Brasil.

\section{Correspondência:}

Francisco Zacaron Werneck. Universidade Federal de Ouro Preto, Rua Dois, 110, Campus Universitário, Ouro Preto - MG, CEP 35400-000. Email: franciscozacaron@yahoo.com.br 


\begin{abstract}
Introduction: Finding children with high sports potential is an important stage in the sports training process, as it is the first step to discover new talents aiming to high performance. The objective was to investigate the determinants of performance in trampoline gymnastics, and Its attributed importance according to discipline apparatuses.

Methodology: Forty trampoline gymnastics experts (coaches, managers and referees) with national and international experience volunteered. The experts answered about the importance given to the anthropometric, physicomotor, technical, tactical, psychological and social-environmental factors. They evaluated the importance of the factors and performance indicators on the acrobatic, tumbling, and double mini trampoline. Results and discussion: The determinants of performance in descent importance order were the physicomotor, technical, psychological, tactical anthropometric and social- environmental factors. Therefore the indicators of performance: muscle mass, speed, agility, flexibility, balance and upper limbs explosive strength have been differently distinguished among the apparatuses. Conclusion: The expert's opinions were revealed about which determinants of performance have greater value in the detection and selection of talents.
\end{abstract}

Keywords: Talent. Youth Sports. Sports Performance.

\title{
RESUMEN
}

Introducción: Encontrar niños con elevado potencial deportivo es una etapa importante en el proceso de formación deportiva, ya que es el primer paso para encontrar nuevos talentos con el objetivo del alto rendimiento deportivo. El objetivo fue investigar los determinantes del rendimiento en la gimnasia de trampolín, e la importancia atribuida de acuerdo con los aparatos de la disciplina. Metodología: Han participado cuarenta expertos en gimnasia en trampolín (entrenadores, gestores y árbitros) con experiencia nacional y internacional. Los expertos respondieron sobre la importancia dada a los factores antropométricos, fisicomotores, técnicos, tácticos, psicológicos y socio-ambientales. Evaluaron la importancia de los factores y los indicadores de rendimiento en el trampolín acrobático, tumbling y el doble mini. Resultados e discusión: Los determinantes del desempeño en orden de importancia decreciente fueron: físico motores, técnicos, psicológicos, tácticos, antropométricos y socio ambientales. Los indicadores de rendimiento, masa muscular, velocidad, agilidad, flexibilidad, equilibrio y fuerza rápida de las extremidades superiores tienen diferencias entre los aparatos. Conclusión: se reveló la opinión de los expertos sobre cuales los determinantes del desempeño son más importantes e de más valor en la detección y selección de talentos en la gimnasia de trampolín.

Palabras Clave: Talento. Deportes Juveniles. Rendimiento Deportivo. 


\section{INTRODUÇÃO}

A ginástica é uma atividade que há muito tempo vem sendo praticada pelo homem, e ao decorrer do seu percurso histórico, vem sofrendo diversas adaptações e transformações. O universo ginástico é amplo, dentre eles: ginástica de condicionamento físico, fisioterápica, de demonstração e as de competição. As ginásticas de competição e demonstração são aquelas organizadas pela Federação Internacional de Ginástica (FIG). A Ginástica Artística (GA) Feminina e Masculina, a Ginástica Rítmica (RG) e a Ginástica de Trampolim (GTR) são modalidades olímpicas, enquanto que a Ginástica Acrobática, a Ginástica Aeróbica, a Ginástica para todos e o Parkour são modalidades não olímpicas (FIG, 2019; TSUKAMOTO; NUNOMURA; CARRARA, 2011).

A GTR é composta por três aparelhos: Trampolim acrobático (TRA), Tumbling (TU) e Duplo Mini-Trampolim (DMT) (FIG, 2017). A GTR é uma modalidade esportiva na qual são realizados saltos acrobáticos sem interrupções, onde o controle corporal, precisão, velocidade e equilíbrio dinâmico são fundamentais. Assim, exige na preparação física o desenvolvimento das capacidades motoras mais importantes como a velocidade, a força e a flexibilidade (MOREIRA; ARAÚJO, 2004). Desde o ano de 2000, com a inclusão do TRA individual nos Jogos Olímpicos, a GTR ganhou mais atenção das autoridades esportivas e vem atraindo investimentos, pesquisadores e mídia (BORTOLETO; CARRARA; ROVERI, 2018). Contudo, ainda é uma modalidade pouco difundida na comunidade científica, e as pesquisas sobre a GTR no Brasil ainda são escassas.

Os campeonatos mundiais por idades na GTR iniciam com categorias a partir dos 11 anos de idade (KUNZE, 2019). Já no Brasil os campeonatos de GTR iniciam com categorias a partir dos nove anos de idade (CBG, 2017). Como na GTR existem poucas pesquisas, em outras modalidades similares, tais como na GA, os técnicos justificam que por conta da cultura da modalidade, e pelas exigências competitivas das entidades organizadoras, a iniciação esportiva começa em tenra idade e as crianças se especializam muito cedo (NUNOMURA; PIRES; CARRARA, 2009). $\mathrm{Na}$ opinião dos treinadores brasileiros, a idade ideal para iniciar a prática esportiva na GA seria entre cinco a sete anos de idade (FONTANA et al., 2014; NUNOMURA; CARRARA; TSUKAMOTO, 2010). Na GTR, em média, a idade de início é aos 7-8 anos, mais tardia em relação às outras modalidades ginásticas, mas ainda assim é precoce comparado a maioria dos esportes (OLIVEIRA, 2018).

Na ginástica, a identificação de crianças e jovens com elevado potencial esportivo tem interessado treinadores, dirigentes, pesquisadores e organizações esportivas, atraindo investimentos em modelos de identificação e desenvolvimento de talentos (ALBUQUERQUE; FARINATTI, 2007). O treinamento em longo prazo, desenvolvido de forma sistematizada e planejada, tem um importante papel na seleção, deteç̧ão e promoção de talentos; e tem como principal objetivo a formação esportiva de atletas para o alto rendimento, desde as categorias de base até o 
esporte de alto nível (BÖHME, 2000). Os determinantes de desempenho no esporte são multifatoriais, pois além das características motoras, físicas, psicológicas, antropométricas e do desempenho em competições, deve-se considerar também o ambiente em que o indivíduo está exposto e o apoio recebido pelos treinadores e familiares (LARKIN; O'CONNOR, 2017; PANKHURST; COLLINS, 2013).

As avaliações na GTR podem ser diferentes de outros esportes por apresentar características específicas da modalidade. Lanaro Filho e Böhme (2001) relatam a importância de seleção, detecção e promoção de talentos na ginástica rítmica. Muitas vezes os atletas não possuem os requisitos necessários para o desempenho de alto rendimento para os esportes que praticam, e isso pode acarretar em anos de treinos sem o desenvolvimento esperado, além da frustração de não atingir os objetivos. Por conta do elevado grau de exigência na ginástica, ressaltamos a importância da seleção de talentos, onde o trabalho para as crianças com grande potencial precisa ser diferenciado das crianças em processo de desenvolvimento (ALBUQUERQUE; FARINATTI, 2007). Esse processo de seleção ajuda também a adequar o planejamento de aulas, a forma mais assertiva para os grupos, evitando uma possível sobrecarga para as crianças. As possibilidades de sucesso dependem dos traços individuais, metodologia de ensino, potencial genético e treinamento adequado durante os estágios de desenvolvimento, além das capacidades mentais e condições ambientais favoráveis (LANARO FILHO; BÖHME, 2001).

Compreender a importância dos determinantes do desempenho esportivo é uma importante tarefa para os pesquisadores e técnicos. Os treinadores experientes são capazes de identificar alunos/atletas com um grande potencial esportivo, assim como a influência e a interação das características indispensáveis para o alto rendimento, características estas relacionadas ao indivíduo, ao ambiente e à tarefa (GREENWOOD; DAVIDS; RENSHAW, 2014). De acordo com o Coaching Model, proposto por Côté e colaboradores (1995), a percepção dos treinadores é baseada em suas experiências e em seu conhecimento. Trata-se de uma importante fonte de informação, pois são eles os principais responsáveis pelas tomadas de decisão relacionadas aos atletas. Os treinadores mais experientes têm uma ideia sobre as habilidades atuais do ginasta e do seu potencial, baseado em comparações com outros ginastas de vários níveis e idades. Assim, a tomada de decisão principalmente relacionada ao sistema de treinamento adequado para o desenvolvimento e evolução do ginasta é talvez das mais importantes fontes de informação para o técnico de ginástica e outros técnicos que buscam o conhecimento e novas experiências (CÔTÉ et al., 1995). Sendo assim, no processo de treinamento a longo prazo do atleta, é importante conhecer os critérios relevantes que os treinadores utilizam para selecionar talentos e compreender a importância atribuída a cada um dos fatores de desempenho (CRIPPS; HOPPER; JOYCE, 2016; PANKHURST; COLLINS, 2013).

Os treinadores da GA feminina, além de ocuparem o cargo de coordenador, são responsáveis por $70 \%$ das tomadas de decisões, resultando no papel 
fundamental na detecção e seleção de ginastas (BACCIOTTI et al., 2019). Essas decisões têm como base sua formação acadêmica, trajetória profissional, vivência no ginásio e experiência como atleta, que é um recurso engrandecedor na sua formação. Em diferentes modalidades esportivas a opinião dos treinadores tem sido determinante para a avaliação do desempenho, sendo investigada, por exemplo, no handebol (MASSUÇA; FRAGOSO, 2010), voleibol (MILLISTETD et al., 2013) futebol (CRIPPS; HOPPER; JOYCE, 2016) e atletismo (AGUIAR, 2018). Porém, em relação à GTR as informações atuais são insuficientes, necessitando de pesquisas de campo sobre esta temática. Apesar da importância da modalidade, pouca atenção foi dada por pesquisadores nacionais e estrangeiros a este esporte (UÇAN, 2018).

Compreender e classificar o grau de importância dos fatores antropométricos, físico-motores, técnicos, táticos, psicológicos e socioambientais na GTR irá contribuir para o entendimento de indicadores relevantes para a performance nesta modalidade, com implicações no processo de treinamento dos ginastas de trampolim, servindo de embasamento para os profissionais da área. Estudo recente do nosso laboratório realizou uma caracterização do perfil de atletas dessa modalidade (OLIVEIRA, 2018). Destaca-se a importância de levar em conta a especificidade de cada tipo de aparelho na avaliação do potencial esportivo de jovens atletas, o que permite traçar um quadro teórico em relação à importância relativa de cada variável para o desempenho. O presente estudo sobre a opinião de experts busca aperfeiçoar este entendimento, revelando, por exemplo, o peso que se deve atribuir aos determinantes do desempenho em modelos de identificação de talentos para a GTR.

Portanto, o objetivo do estudo foi investigar a importância atribuída por experts (treinadores, árbitros e gestores) às variáveis determinantes do desempenho na GTR, considerando as diferenças entre os tipos de aparelhos da modalidade.

\section{MÉTODO}

Este estudo é parte integrante do "Projeto Atletas de Ouro: Avaliação Multidimensional e Longitudinal do Potencial Esportivo de Jovens Atletas", aprovado pelo Comitê de Ética em Pesquisa com Seres Humanos da Universidade Federal de Ouro Preto (CAAE: 32959814.4.1001.5150), que tem por finalidade construir uma modelagem para identificação e desenvolvimento de talentos esportivos (WERNECK et al., 2017a).

Participaram do estudo 40 experts de GTR, sendo 22 mulheres e 18 homens, com média de idade de 34,9 $\pm 12,0$ anos, e tempo de experiência média de 13,6 \pm 6,7 anos. O consentimento dos treinadores, gestores e árbitros, foram obtidos durante o congresso técnico da competição. Como critério de inclusão adotou-se ser árbitro, ex-atleta que seja árbitro ou assistente técnico e treinador de ginástica de 
trampolim. A coleta dos dados foi realizada durante os Campeonatos Estadual e Brasileiro de GTR em Ouro Preto, Minas Gerais, nos anos de 2018 e 2019.

Os experts voluntariamente responderam um questionário para avaliação dos determinantes do desempenho esportivo, adaptado a partir de estudos anteriores realizados em outras modalidades (LARKIN, O'CONNOR, 2017; MAÇUSSA, FRAGOSO, 2010) e levando-se em conta os fatores e indicadores de avaliação do potencial esportivo de jovens atletas que compõem a bateria de testes do Projeto Atletas de Ouro ${ }^{\circledR}$ - uma pesquisa longitudinal que tem por finalidade a construção de um modelo de identificação de talentos esportivos (WERNECK et al., 2017b). Portanto, o conteúdo e o delineamento do questionário foi criado tomando como base a literatura científica sobre identificação e desenvolvimento de talentos esportivos (BACCIOTTI et al., 2019; PANKHURST; COLLINS, 2013) e a necessidade de obtenção de informação específica para as variáveis coletadas no Projeto Atletas de Ouro $\Re$. Estudo realizado pelo nosso grupo de pesquisa com treinadores de atletismo demonstrou coerência entre a opinião dos treinadores e a evidência científica acerca da importância das variáveis para a performance na modalidade (AGUIAR, 2018).

Na primeira parte da presente pesquisa, constaram informações demográficas e experiência profissional. Na segunda parte, os experts responderam sobre a importância atribuída a cada um dos seguintes fatores de desempenho: 1) antropométrico; 2) físico-motor; 3) técnico; 4) tático; 5) psicológico e 6) socioambiental, utilizando uma escala Lickert de cinco pontos (1-nada importante, 2-pouco importante, 3-importante, 4-muito importante e 5-extremamente importante). Em seguida, classificaram de 1 a 6, a ordem de importância de cada um destes fatores para o desempenho na ginástica de trampolim de uma maneira geral, sendo um (1) o mais importante e seis (6) o menos importante.

$\mathrm{Na}$ terceira parte do questionário, os fatores de desempenho foram estratificados em 47 indicadores, e os treinadores deveriam atribuir a importância a cada um deles para os diferentes tipos de aparelhos. 1) Fator antropométrico (8 indicadores): peso, altura, envergadura, massa muscular, comprimento dos membros inferiores, tamanho do tronco, largura dos ombros e tamanho da mão/pé; 2) Fator fisicomotor (12 indicadores): velocidade, agilidade, força/potência muscular de membros inferiores, tempo de reação, flexibilidade, equilíbrio, acuidade visual, potência/resistência aeróbica, potência/resistência anaeróbica, força de preensão manual, força/potência muscular de membros superiores e lateralidade; 3) Fator técnico (5 indicadores): habilidades locomotoras, habilidades manipulativas, habilidades estabilizadoras, habilidades motoras específicas, eficiência/qualidade de execução do movimento; 4) Fator tático (5 indicadores): capacidade de antecipação, capacidade de tomada de decisão, habilidade de usar boas estratégias, inteligência motora e pensamento criativo; 5) Fator psicológico (11 indicadores): espírito de equipe, autoconfiança, preparação mental, Capacidade de suportar pressão, atenção/concentração, competitividade, motivação, saber lidar 
com adversidades, treinabilidade, liderança e estabilidade emocional; 6) Fator socioambiental ( 5 indicadores): história atlética dos pais, nível socioeconômico, rendimento escolar, apoio familiar e experiência esportiva.

\section{ANÁLISE EstATÍSTICA}

Para a análise dos dados, utilizou-se a estatística descritiva [Moda, Mediana (10quartil - $3^{\circ}$ quartil) para caracterizar a importância atribuída aos fatores e indicadores de desempenho, além do intervalo de confiança de 95\% (IC95\%) da média. Para detectar diferenças entre as modalidades em relação aos indicadores e fatores de desempenho, utilizou-se a ANOVA de Friedman, seguida pelo teste de Wilcoxon para comparação pareada. Todas as análises foram feitas no software IBM SPSS versão 24.0 (IBM Corp., Armonk, NY). O valor de $\mathrm{p} \leq 0,05$ foi adotado para significância estatística.

\section{Resultados}

\section{CARACTERÍSTICAS dOS PARTICIPANTES}

As características dos participantes quanto ao gênero, Estado federativo, função, histórico de atleta e nível competitivo são apresentadas na Tabela 1.

Tabela 1 - Característica dos participantes

\begin{tabular}{cc}
\hline \multicolumn{1}{c}{ Variáveis } & N (\%) \\
Gênero & $22(55 \%)$ \\
Feminino & $18(45 \%)$ \\
Masculino & \\
Estado federativo & $30(75 \%)$ \\
Minas Gerais & $6(15 \%)$ \\
Rio de Janeiro & $3(7,5 \%)$ \\
São Paulo & $1(2,5 \%)$ \\
Rio Grande do Sul & \\
Função atual & $20(50 \%)$ \\
Treinador & $3(7,5 \%)$ \\
Pesquisador & $2(5,0 \%)$ \\
Gestor & $15(37,5 \%)$ \\
Ex-atleta & $11(27,5 \%)$ \\
Histórico de atleta & $29(72,5 \%)$ \\
Não & $1(2,5 \%)$ \\
Sim & $7(17,5 \%)$ \\
Nível competitivo & $14(35 \%)$ \\
Estadual & Nacional \\
Internacional &
\end{tabular}

IMPORTÂNCIA ATRIBUÍDA AOS DETERMINANTES DO DESEMPENHO 
Na opinião dos experts os determinantes de desempenho que possuem maior grau de importância na GTR são os fatores fisicomotores e técnicos, seguidos dos fatores psicológicos e socioambientais, e por fim os fatores táticos e antropométricos $\left(X^{2}=87,138 ; g l=5 ; p<0,001\right)$. A ordem de importância sobre os fatores de desempenho segundo os treinadores são: 10 Físico-motores; 20 Técnicos; 30 Psicológicos; 40 Táticos; 50 Antropométrico; e 60 Socioambientais $\left(X^{2}=94,411 ; g l=5 ; p<0,001\right)-$ Tabela 2 .

Tabela 2 - Grau e ordem de importância atribuída pelos experts aos fatores do desempenho na GTR $(n=40)$

\begin{tabular}{|c|c|c|c|c|c|c|}
\hline \multirow{2}{*}{$\begin{array}{l}\text { Fatores de } \\
\text { Desempenho }\end{array}$} & \multicolumn{3}{|c|}{ Grau de Importância* } & \multicolumn{3}{|c|}{ Ordem de Importância** } \\
\hline & Moda & $\begin{array}{c}\text { Mediana } \\
\left(1^{\circ} \mathrm{Q}-3^{\circ} \mathrm{Q}\right)\end{array}$ & Soma & Moda & $\begin{array}{c}\text { Mediana } \\
\left(1^{\circ} \mathrm{Q}-3^{\circ} \mathrm{Q}\right)\end{array}$ & Soma \\
\hline Físico-motores & 5,0 & $\begin{array}{c}5,0 \\
(5,0-5,0)\end{array}$ & 169 & 10 & $\begin{array}{c}2^{0} \\
\left(1^{0}-2^{\circ}\right)\end{array}$ & 72 \\
\hline Técnicos & 5,0 & $\begin{array}{c}5,0 \\
(5,0-5,0)\end{array}$ & 167 & 10 & $\begin{array}{c}2^{\circ} \\
\left(1^{0}-3^{\circ}\right)\end{array}$ & 77 \\
\hline Psicológicos & 5,0 & $\begin{array}{c}5,0 \\
(4,0-50)\end{array}$ & 159 & 30 & $\begin{array}{c}3^{0} \\
\left(2,5^{0}-4^{\circ}\right)\end{array}$ & 120 \\
\hline Táticos & 4,0 & $\begin{array}{c}4,0 \\
(3,0-4,0)\end{array}$ & 124 & 30 & $\begin{array}{c}5^{0} \\
\left(4^{0}-6^{0}\right)\end{array}$ & 181 \\
\hline Antropométricos & 3,0 & $\begin{array}{c}4,0 \\
(3,0-5,0)\end{array}$ & 131 & 50 & $\begin{array}{c}4^{0} \\
\left(3^{\circ}-5^{\circ}\right)\end{array}$ & 145 \\
\hline Socioambientais & 4,0 & $\begin{array}{c}4,0 \\
(3,0-4,0)\end{array}$ & 129 & 60 & $\begin{array}{c}5^{0} \\
\left(4^{0}-6^{\circ}\right)\end{array}$ & 186 \\
\hline
\end{tabular}

*Grau de importância: 1-nada importante até 5-extremamente importante.

**Ordem de importância: Do $1^{\circ}$ mais importante até o $6^{\circ}$ mais importante.

COMPARAÇÃo DA IMPORTÂNCIA ATRIBUÍDA aos determinantes do desempenho

Ao considerar os fatores determinantes para cada uma das provas, no DMT o fator tático teve maior grau de importância em relação aos outros aparelhos. No TRA o maior grau de importância atribuído foi aos fatores técnicos. No TU foi classificado como mais importantes os fatores antropométricos e físico-motores, comparados aos aparelhos TRA e DMT. Já. Os fatores psicológicos, socioambientais e táticos não apresentaram diferenças significativas entre os grupos de provas. 


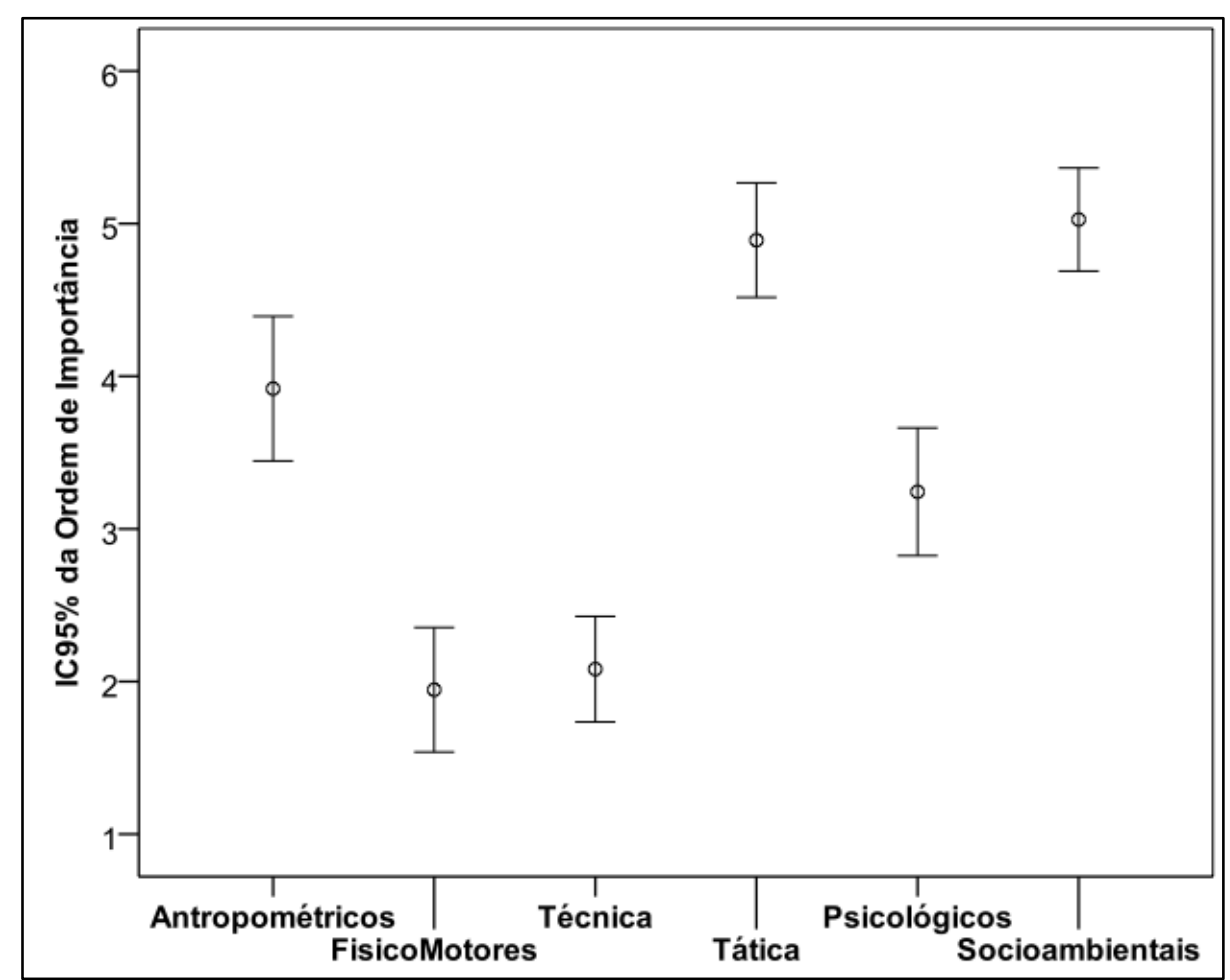

Figura 1 - Intervalo de confiança de 95\% para a média da ordem de importância atribuída pelos experts aos fatores do desempenho na ginástica de trampolim $(n=40)$.

*Ordem de importância: Do $1^{\circ}$ mais importante (1) até o $6^{\circ}$ mais importante (6).

Tabela 3 - Importância atribuída pelos experts aos fatores do desempenho na GTR $(n=40)$ em função do aparelho

\begin{tabular}{cccccc}
\hline $\begin{array}{c}\text { Fatores de } \\
\text { Desempenho }\end{array}$ & Duplo Mini & $\begin{array}{c}\text { Aparelho } \\
\text { Trampolim }\end{array}$ & Tumbling & $\mathbf{X}^{\mathbf{2}}$ & p-valor \\
\hline Técnico & 5,0 & 5,0 & 5,0 & 15,83 & $<0,001$ \\
& $(4,0-5,0)$ & $(5,0-5,0)$ & $(4,0-5,0)$ & 0 & $*$ \\
Antropométrico & 3,5 & 4,0 & 4,0 & 14,74 & $0,001^{*}$ \\
& $(3,0-5,0)$ & $(3,0-4,75)$ & $(3,0-5,0)$ & 7 & \\
Físico-motor & 5,0 & 4,5 & 5,0 & 5,633 & 0,06 \\
& $(4,0-5,0)$ & $(4,0-5,0)$ & $(4,0-5,0)$ & & \\
Tático & 4,0 & 4,0 & 4,0 & 2,667 & 0,26 \\
Psicológico & $(3,0-5,0)$ & $(3,0-4,0)$ & $(3,0-4,0)$ & & \\
& 5,0 & 5,0 & 5,0 & 2,400 & 0,30 \\
Socioambiental & $(4,0-5,0)$ & $(4,0-5,0)$ & $(4,0-5,0)$ & & \\
& $(3,0-4,0)$ & $(3,0-4,0)$ & $(3,0-4,0)$ & & \\
\hline
\end{tabular}

Legenda: Mediana $\left(1^{\circ} \mathrm{Q}-3^{\circ} \mathrm{Q}\right)$; 1-nada importante até 5-extremamente importante.

*diferença estatisticamente significativa, $\mathrm{p}<0,05$. 


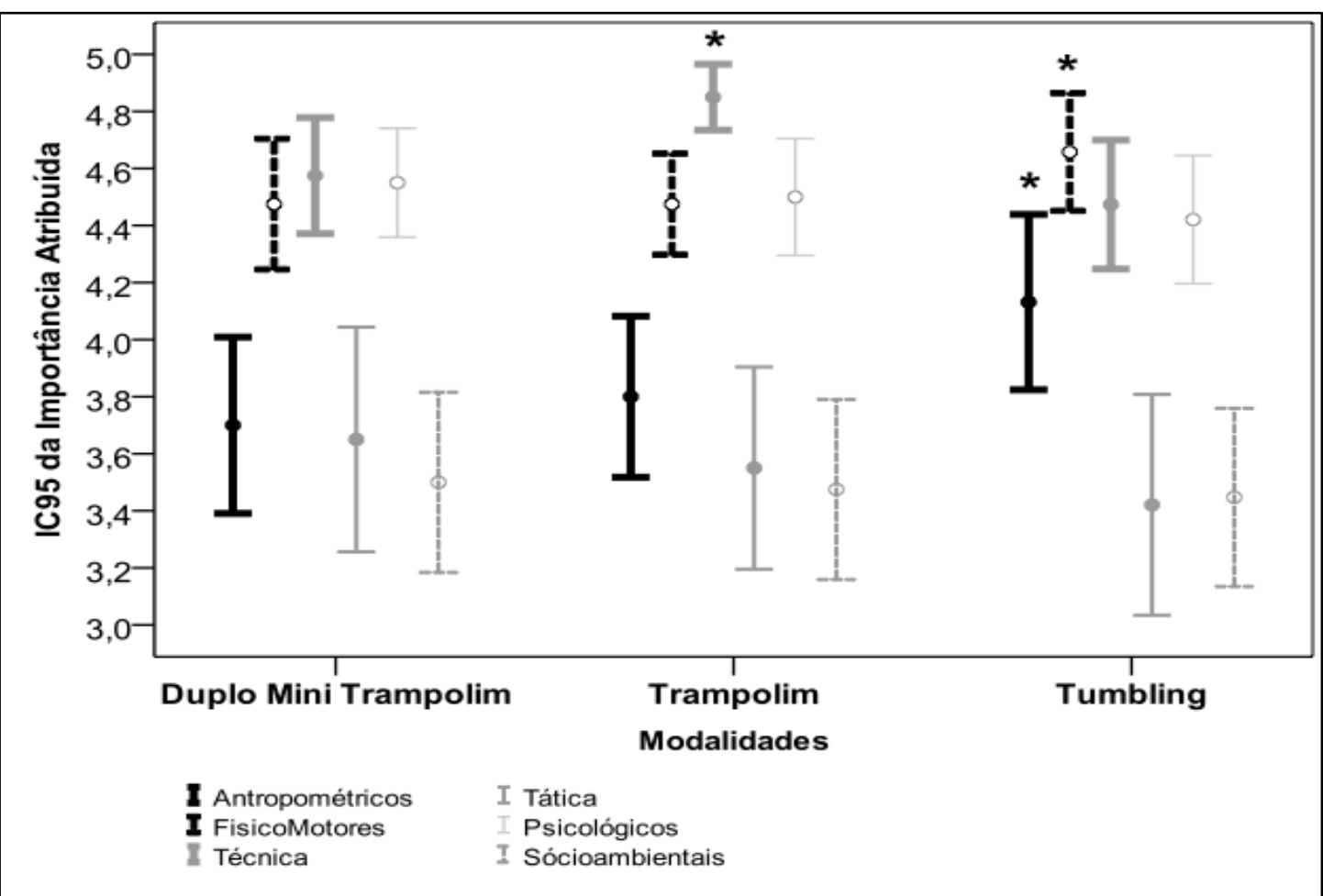

Figura 2 - Intervalo de confiança de 95\% para a média de importância na comparação das diferentes modalidades na GTR $(n=40)$. (1-nada importante até 5-extremamente importante; *diferença estatisticamente significativa, $\mathrm{p}<0,05)$.

Os indicadores de desempenho que apresentaram diferença significativa entre os aparelhos foram: no antropométrico a massa muscular $(p=0,01)$, no fisicomotor a velocidade $(p<0,001)$, agilidade $(p=0,02)$, flexibilidade $(p=0,02)$, equilíbrio $(p=0,046)$ e a força rápida de membros superiores $(p=0,01)$. 0 indicador técnico de eficiência e qualidade de movimento ficou próximo ao nível de significância $(p=0,07)$.

Tabela 4 - Importância atribuída pelos experts $(n=40)$ aos indicadores do desempenho na GTR em função do aparelho

\begin{tabular}{|c|c|c|c|c|c|}
\hline \multirow{2}{*}{$\begin{array}{c}\text { Fatores / } \\
\text { Indicadores }\end{array}$} & \multicolumn{3}{|c|}{ Aparelho } & \multirow{2}{*}{$\mathbf{x}^{2}$} & \multirow{2}{*}{$\begin{array}{c}\text { p- } \\
\text { valor }\end{array}$} \\
\hline & Duplo Mini & Trampolim & Tumbling & & \\
\hline \multicolumn{6}{|l|}{ Antropométrico } \\
\hline Massa muscular & $3,0(4,0-5,0)$ & $3,0(4,0-4,0)$ & $4,0(5,0-5,0)$ & 9,110 & $0,01^{*}$ \\
\hline Largura dos ombros & $2,0(2,0-3,0)$ & $2,5(2,0-3,7)$ & $3,0(2,0-4,0)$ & 2,197 & 0,33 \\
\hline Altura & $3,0(2,0-4,0)$ & $3,0(2,0-4,0)$ & $3,0(2,0-4,0)$ & 1,213 & 0,54 \\
\hline Comp de mmii & $3,0(2,0-4,0)$ & $3,0(2,5-4,0)$ & $3,0(2,0-4,0)$ & 1,062 & 0,59 \\
\hline Peso & $4,0(3,2-4,0)$ & $4,0(4,0-5,0)$ & $4,0(3,7-5,0)$ & 0,678 & 0,71 \\
\hline Tamanho do Tronco & $3,0(2,0-4,0)$ & $3,0(2,0-4,0)$ & $3,0(2,0-4,0)$ & 0,690 & 0,71 \\
\hline Tamanho da mão/pé & $2,0(1,0-3,0)$ & $2,0(2,0-3,0)$ & $2,0(1,0-3,0)$ & 0,478 & 0,79 \\
\hline Envergadura & $3,0(2,0-3,0)$ & $3,0(2,0-4,0)$ & $3,0(2,0-4,0)$ & 0,442 & 0,80 \\
\hline
\end{tabular}




\section{Fisicomotor}

\begin{tabular}{|c|c|c|c|c|c|}
\hline Velocidade & $5,0(4,0-5,0)$ & $4,0(2,0-4,0)$ & $5,0(4,0-5,0)$ & 25,98 & $\begin{array}{l}<0,00 \\
1^{*}\end{array}$ \\
\hline $\begin{array}{l}\text { Força/potência } \\
\text { muscular mmss }\end{array}$ & $3,0(2,0-4,0)$ & $3,0(2,0-4,0)$ & $4,0(3,0-5,0)$ & 9,705 & $0,01^{*}$ \\
\hline Agilidade & $5,0(4,0-5,0)$ & $4,0(3,0-5,0)$ & $5,0(4,0-5,0)$ & 8,047 & $0,02 *$ \\
\hline Flexibilidade & $4,0(3,0-5,0)$ & $5,0(4,0-5,0)$ & $3,0(3,0-4,2)$ & 7,970 & $0,02 *$ \\
\hline Equilíbrio & $5,0(4,0-5,0)$ & $4,5(4,0-5,0)$ & $4,0(3,0-5,0)$ & 6,156 & $0,046^{*}$ \\
\hline $\begin{array}{l}\text { otência/Resistência } \\
\text { aeróbica }\end{array}$ & $5,0(4,0-5,0)$ & $4,0(3,0-5,0)$ & $5,0(3,0-5,0)$ & 3,955 & 0,14 \\
\hline Acuidade Visual & $3,0(3,0-4,0)$ & $4,0(3,0-5,0)$ & $3,0(3,0-4,0)$ & 3,253 & 0,20 \\
\hline $\begin{array}{l}\text { Força/potência } \\
\text { muscular mmii }\end{array}$ & $5,0(4,0-5,0)$ & $5,0(4,0-5,0)$ & $5,0(5,0-5,0)$ & 2,927 & 0,23 \\
\hline $\begin{array}{l}\text { Potência/Resistência } \\
\text { anaeróbica }\end{array}$ & $5,0(4,0-5,0)$ & $4,0(4,0-5,0)$ & $5,0(4,0-5,0)$ & 2,221 & 0,33 \\
\hline $\begin{array}{l}\text { Força de preensão } \\
\text { manual }\end{array}$ & $2,0(1,0-3,0)$ & $2,0(1,0-3,7)$ & $2,0(1,7-4,0)$ & 1,636 & 0,44 \\
\hline Lateralidade & $3,0(2,0-5,0)$ & $3,0(2,0-5,0)$ & $3,0(2,0-4,0)$ & 0,307 & 0,86 \\
\hline $\begin{array}{c}\text { Tempo de reação } \\
\text { Técnico }\end{array}$ & $5,0(4,0-5,0)$ & $5,0(4,0-5,0)$ & $5,0(4,0-5,0)$ & 0,136 & 0,93 \\
\hline $\begin{array}{l}\text { Eficiência/Qualidade } \\
\text { de movimento }\end{array}$ & $5,0(5,0-5,0)$ & $5,0(5,0-5,0)$ & $5,0(4,0-5,0)$ & 5,226 & 0,07 \\
\hline $\begin{array}{l}\text { Habilidades motoras } \\
\text { específicas }\end{array}$ & $5,0(5,0-5,0)$ & $5,0(5,0-5,0)$ & $5,0(4,0-5,0)$ & 2,357 & 0,31 \\
\hline $\begin{array}{l}\text { Habilidades } \\
\text { locomotoras }\end{array}$ & $5,0(4,2-5,0)$ & $5,0(4,0-5,0)$ & $5,0(4,0-5,0)$ & 0,417 & 0,81 \\
\hline $\begin{array}{l}\text { Habilidades } \\
\text { estabilizadoras }\end{array}$ & $5,0(4,2-5,0)$ & $5,0(4,2-5,0)$ & $5,0(4,0-5,0)$ & 0,40 & 0,98 \\
\hline $\begin{array}{c}\text { Habilidades } \\
\text { manipulativas } \\
\text { Tático }\end{array}$ & $2,0(1,0-3,0)$ & $2,0(1,0-3,0)$ & $2,0(1,0-3,5)$ & 0,040 & 0,98 \\
\hline $\begin{array}{l}\text { Capacidade de } \\
\text { tomada de decisão }\end{array}$ & $4,0(3,0-5,0)$ & $4,5(4,0-5,0)$ & $4,0(3,5-5,0)$ & 0,702 & 0,70 \\
\hline $\begin{array}{l}\text { Habilidade de usar } \\
\text { boas estratégias }\end{array}$ & $4,0(3,0-5,0)$ & $4,0(3,0-5,0)$ & $4,0(3,0-5,0)$ & 0,544 & 0,76 \\
\hline Inteligência motora & $5,0(4,0-5,0)$ & $5,0(4,0-5,0)$ & $5,0(4,0-5,0)$ & 0,393 & 0,82 \\
\hline $\begin{array}{l}\text { Capacidade de } \\
\text { antecipação }\end{array}$ & $4,0(3,0-5,0)$ & $4,0(3,0-5,0)$ & $4,0(3,0-5,0)$ & 0,344 & 0,84 \\
\hline Pensamento criativo & $3,0(2,0-4,0)$ & $3,0(2,0-4,0)$ & $3,0(2,0-4,5)$ & 0,037 & 0,98 \\
\hline
\end{tabular}




\begin{tabular}{|c|c|c|c|c|c|}
\hline \multicolumn{6}{|l|}{ Psicológico } \\
\hline Autoconfiança & $5,0(5,0-5,0)$ & $5,0(4,0-5,0)$ & $5,0(4,5-5,0)$ & 0,047 & 0,098 \\
\hline $\begin{array}{c}\text { Atenção/ } \\
\text { Concentração }\end{array}$ & $5,0(5,0-5,0)$ & $5,0(5,0-5,0)$ & $5,0(5,0-5,0)$ & 1,488 & 0,47 \\
\hline Competitividade & $4,0(4,0-5,0)$ & $4,0(4,0-5,0)$ & $4,0(4,0-4,2)$ & 1,370 & 0,50 \\
\hline Liderança & $3,0(2,0-4,0)$ & $3,0(3,0-4,0)$ & $3,0(2,0-4,0)$ & 1,142 & 0,56 \\
\hline Treinabilidade & $5,0(4,0-5,0)$ & $5,0(4,0-5,0)$ & $5,0(4,0-5,0)$ & 0,750 & 0,69 \\
\hline $\begin{array}{l}\text { Estabilidade } \\
\text { emocional }\end{array}$ & $4,5(4,0-5,0)$ & $4,0(4,0-5,0)$ & $4,0(4,0-5,0)$ & 0,283 & 0,87 \\
\hline $\begin{array}{l}\text { Capacidade de } \\
\text { suportar pressão }\end{array}$ & $5,0(4,0-5,0)$ & $5,0(4,0-5,0)$ & $5,0(4,0-5,0)$ & 0,163 & 0,92 \\
\hline Motivação & $5,0(4,0-5,0)$ & $5,0(4,0-5,0)$ & $5,0(4,0-5,0)$ & 0,087 & 0,96 \\
\hline Preparação Mental & $5,0(5,0-5,0)$ & $5,0(5,0-5,0)$ & $5,0(5,0-5,0)$ & 0,071 & 0,96 \\
\hline $\begin{array}{l}\text { Saber lidar com } \\
\text { adversidades }\end{array}$ & $4,0(4,0-5,0)$ & $4,0(4,0-5,0)$ & $4,0(4,0-5,0)$ & 0,071 & 0,96 \\
\hline Espírito de equipe & $4,0(3,0-5,0)$ & $4,0(3,0-5,0)$ & $4,0(3,0-5,0)$ & 0,011 & 0,99 \\
\hline \multicolumn{6}{|l|}{ Socioambiental } \\
\hline $\begin{array}{c}\text { Nível } \\
\text { socioeconômico }\end{array}$ & $3,0(2,0-3,0)$ & $3,0(2,0-3,0)$ & $2,0(2,0-3,0)$ & 1,106 & 0,57 \\
\hline Apoio familiar & $4,0(3,0-5,0)$ & $4,0(3,0-5,0)$ & $4,0(3,0-5,0)$ & 0,115 & 0,94 \\
\hline endimento escolar & $3,0(2,0-4,0)$ & $3,0(2,2-4,0)$ & $3,0(2,0-4,0)$ & 0,043 & 0,98 \\
\hline $\begin{array}{l}\text { Experiência } \\
\text { esportiva }\end{array}$ & $3,0(3,0-4,0)$ & $3,0(3,0-4,0)$ & $3,0(3,0-4,0)$ & 0,026 & 0,99 \\
\hline $\begin{array}{c}\text { istória atlética dos } \\
\text { pais }\end{array}$ & $2,0(3,0-3,0)$ & $2,0(2,0-3,0)$ & $2,0(2,0-3,0)$ & 0,016 & 0,99 \\
\hline
\end{tabular}

Legenda: Mediana $\left(1^{\circ} \mathrm{Q}-3^{\circ} \mathrm{Q}\right)$.

\section{DISCUSSÃo}

O presente estudo teve como objetivo identificar qual a importância atribuída pelos experts a cada uma das variáveis determinantes do desempenho na GTR, sendo elas divididas em antropométricas, físico-motoras, técnicas, táticas, psicológicas e socioambientais, como também analisar as diferenças entre os três aparelhos da modalidade (TRA, TU, DMT).

Visto que o acervo científico na GTR carece de estudos sobre o papel dos treinadores no desempenho dos atletas (PANKHURST; COLLINS, 2013), não encontramos literatura sobre a importância dos determinantes de desempenho especificamente na modalidade de GTR. A opinião dos técnicos vem sendo atualmente a resposta mais frequente para a detecção e identificação de talentos 
(BACCIOTTI et al., 2019), pois o treinador consegue identificar o potencial esportivo necessário para a modalidade (LARKIN; O'CONNOR, 2017). No entanto, o ponto de vista do voluntário acarreta um processo subjetivo quanto aos critérios adotados por esses indivíduos na identificação e seleção destes atletas (PANKHURST; COLLINS, 2013). O presente artigo acrescenta a informação de quem vivenciou a modalidade desde atleta, com 72,5\% de ex-atletas, sendo 53\% de nível competitivo nacional ou internacional.

As características das provas de GTR são distintas: no TRA são realizados 10 saltos acrobáticos ininterruptos, e os critérios de avaliação incluem o tempo de voo (altura do salto) que é somado às notas de execução, dificuldade e deslocamento horizontal (FIG, 2017). Assim, a precisão e o refinamento técnico são indispensáveis neste aparelho onde a área de salto sem penalização é de cerca de $1 \mathrm{~m}^{2}$ (FIG, 2020). No TU, são executados oito elementos em uma pista de $25 \mathrm{~m}$ de comprimento, o que exige força rápida, velocidade e potência. Já no DMT a velocidade da corrida que antecede o salto é de grande valência, para realizar a série pelo comprimento do aparelho até a área de aterrissagem que está a 3,5m de distância; além da precisão e agilidade para realizar dois a três saltos em uma tela 0,90m de largura (FIG, 2020).

Na opinião dos experts os fatores físico-motores foram considerados os mais importantes, o que corrobora a importância das capacidades físicas e motoras para o desempenho na GTR. A realização de uma rotina na GTR exige determinado nível de flexibilidade, força, equilíbrio (estático e dinâmico), agilidade, coordenação e orientação espacial. Estudo realizado com atletas poloneses de ginástica de trampolim demonstrou que o perfil de aptidão física destes atletas é similar aos de atletas de ginástica acrobática, com destaque para utilização de uma bateria de testes multidimensional, que avalie as principais capacidades físicas exigidas na modalidade (SEREDYŃSKI; POLAK, 2015). A força, por exemplo, é um fator indispensável no desempenho humano (ZATSIORSKY; KRAEMER, 2006). Na GTR, a força muscular apresenta maior importância no TU, que possui movimentos similares aos do solo na GA (NUNOMURA; PIRES; CARRARA, 2009).

Os fatores técnicos foram classificados como o segundo conjunto mais importante, evidenciando maior grau de importância no TRA, onde a técnica é indispensável para uma excelente execução dos movimentos. Por conta da complexidade e especificidade dos movimentos a técnica é essencial, sendo uma característica predominante da Ginástica (BOMPA, 2002). A literatura disponível sobre GTR carece de informações sobre o desempenho técnico dos atletas, uma vez que os pesquisadores têm dedicado maior atenção aos aspectos relacionados a aptidão física (SEREDYŃSKI; POLAK, 2015) e ao perfil antropométrico e de composição corporal (SIAHKOUHIAN; AALIZADEH; ESMAEILZADE, 2013). Para além da execução perfeita do movimento visando a performance competitiva, destaca-se a importância da técnica na prevenção de lesões, haja visto que com a evolução de técnicas e rotinas mais competitivas, o potencial de lesões graves em 
competição também aumenta (ESPOSITO; ESPOSITO, 2009). Sendo assim, a técnica deve ser continuamente aprendida e treinada, respeitando as etapas de formação dentro do processo de treinamento a longo prazo, evitando queimar etapas (NUNOMURA; CARRARA; TSUKAMOTO, 2010; TSUKAMOTO; NUNOMURA; CARRARA, 2011).

Os fatores antropométricos e físico-motores são considerados mais importantes no TU, corroborando os nossos estudos, autores afirmam que os ginastas mais baixos e fortes têm mais potência para implementar habilidades mais complexas que envolvem todo o corpo (FARIA; FARIA, 1989; UÇAN, 2018), justificando o perfil dos atletas de TU. Cerca de $45 \%$ dos fatores antropométricos estão envolvidos no desempenho ginástico (DOUDA et al., 2008). No TR a maior proporção dos atletas são endomórficos (UÇAN, 2018), enquanto que a mesomorfia caracteriza os atletas de TU (WERNECK et al., 2017b). Os atletas de GTR têm menor estatura e menor peso corporal que os praticantes de outros esportes (SEREDYŃSKI; POLAK, 2015). Os fatores genéticos e a seleção natural do esporte são os principais motivos para as estaturas dos ginastas (BACCIOTTI et al., 2018; LANARO FILHO; BÖHME, 2001; NUNOMURA; CARRARA; TSUKAMOTO, 2010).

Entre os indicadores de desempenho que apresentaram diferença significativa nos diferentes aparelhos, a massa muscular apresentou maior importância no TU, onde normalmente observam-se atletas mais fortes e baixos, porque este padrão antropométrico possibilita maior eficiência mecânica e potência na execução dos movimentos exigidos neste aparelho (CARR, 1997). A mesomorfia apresentou-se como variável discriminante na comparação de atletas de GTR medalhistas e não medalhistas no TU (WERNECK et al., 2017b). De acordo com os resultados obtidos pelo nosso grupo de pesquisa, os atletas medalhistas no TU apresentaram maior força muscular e potência de membros inferiores. Além disso, verificou-se também que os atletas de elevado potencial esportivo, avaliados por seus treinadores, apresentavam maior massa magra, força e potência muscular e maturação biológica avançada (WERNECK et al., 2017b). Colaborando com esta ideia, Uçan (2018) afirma que os atletas de GTR que estão no alto rendimento têm maior massa muscular e menor porcentagem de gordura, além de apresentar maior força e menor estatura. Oliveira (2018) observou que quando comparado aos atletas de GA masculino, os atletas de GTR apresentam menor massa corporal. Provavelmente a maior massa corporal na GA masculina ocorre devido às características específicas da modalidade, que contém maior número de aparelhos com predominância de movimentos sobre os membros superiores.

A flexibilidade apresentou diferença no TRA dos demais aparelhos. A amplitude de movimento é indispensável para facilitar a sua execução (BOMPA, 2002). A flexibilidade é essencial para a boa execução dos movimentos, pois contribui com a qualidade técnica e estética dos elementos (MOREIRA; ARAÚJO, 
2004). Os atletas mais flexíveis na GTR são ectomorfos (UÇAN, 2018). Oliveira (2018) relata que os ginastas na GTR são mais flexíveis na categoria pré-infantil do que nas categorias infantil e infanto-juvenil. Mas comparados com os atletas de GA masculina (FONTANA et al., 2014), os níveis de flexibilidades são mais elevados na GTR (OLIVEIRA, 2018), como também quando comparado a jovens atletas de outras modalidades (MALINA; BOUCHARD, 2002; SEREDYŃSKI; POLAK, 2015).

No DMT o indicador de desempenho equilíbrio foi significativamente mais importante que nos outros aparelhos. Isto pode ocorrer devido à característica do aparelho, onde o ginasta realiza dois ou três saltos em uma tela com largura de 70 centímetros (FIG, 2017). Uçan (2018) ressalta a necessidade do equilíbrio na ginástica, onde determinados aparelhos com largura reduzida requerem muita estabilidade corporal. O equilíbrio em atletas de GTR é tão importante quanto nas demais modalidades de ginástica (HIRATA; OLIVEIRA, 2015). Ao equiparar o equilíbrio estático e dinâmico de mulheres atletas de ginástica, futebol e basquete e observou-se que as atletas de ginástica e futebol têm menores valores de oscilação, o que representa menos desequilíbrio (BRESSEL et al., 2007).

Segundo os experts do presente estudo a força rápida de membros superiores é de grande importância no TU, maior importância se comparado ao TRA e DMT. Ginastas com maior força e potência muscular tem o nível de desempenho mais alto (UÇAN, 2018). No TRA ainda Uçan (2018) explica que para realizar movimentos com eficiência e com maiores valores de dificuldades requer maior rotação para frente e para trás, exigindo a contribuição dos membros superiores.

No fator de desempenho agilidade, os aparelhos DMT e TU foram considerados mais importantes do que o TRA. Isso pode ser explicado pela necessidade de deslocamento do centro de gravidade dos saltos de forma eficaz nestes dois aparelhos (DMT e TU), além do movimento de corrida em alta velocidade necessária para a realização dos movimentos nestes dois aparelhos. Conceitualmente, a agilidade é a capacidade de executar movimentos ligeiros e rápidos com mudanças de direção (BOMPA, 2002). No DMT o tempo de reação é fundamental, considerando a necessidade de realizar o movimento de impulsão em duas situações diferentes: na área de entrada (mount) vindo da corrida de aproximação; e na área de saída (dismount), vindo do primeiro salto. Já os outros dois elementos da agilidade citados acima são importantes no tumbling, onde são necessários movimentos com velocidade para o aumento da nota de dificuldade das séries e amplos para maior nota de execução. No TRA, pelo contrário, deslocarse do centro do aparelho implica em redução da nota do ginasta (FIG, 2017).

Já a velocidade incorpora três elementos: tempo de reação, frequência de movimento por unidade de tempo e velocidade de transposição de uma determinada distância (BOMPA, 2002). Estes elementos estão diretamente 
relacionados com o DMT e o TU, que tem menor elasticidade que o TRA, portanto exigindo do ginasta a execução dos movimentos com menos tempo de fase aérea dos saltos. A velocidade no DMT e TU obteve valores maiores que do TRA, acreditamos que isso seja em decorrência das características de cada aparelho, os dois com maior grau de importância devido à corrida de aproximação que antecede o início dos movimentos, a velocidade e o tipo de passada na pista precisa ser adequada e de forma eficiente, para quando chegar ao aparelho essa transferência de velocidade horizontal em vertical seja suficiente para a execução dos saltos (CARR, 1997). Com isso, a velocidade tem grande valência, pois ela implica no sucesso de execução dos saltos. Já no TRA é necessária a estabilidade no centro do trampolim para a realização dos movimentos sem penalizações (FIG, 2017). Vale ressaltar que os indicadores eficiência e qualidade de movimento ficaram bem próximos ao nível de significância.

Os resultados desse estudo têm implicação no processo de detecção, seleção e desenvolvimento de talentos na GTR. O entendimento dos fatores e indicadores relevantes para a performance na GTR, a partir do conhecimento dos treinadores, é uma importante etapa na construção de protocolos de avaliação do potencial esportivo de jovens ginastas promissores. Esta informação pode ser utilizada nos modelos de identificação de talentos, orientando o jovem atleta para o aparelho mais compatível com o seu perfil. O técnico tem como finalidade desenvolver e explorar as principais qualidades dos atletas, alcançando seu melhor desempenho esportivo. $O$ conhecimento dos treinadores mensurado no presente estudo pode servir de referência de quais indicadores trabalhar de maneira específica para cada grupo de prova e assim, alcançar os objetivos do treinamento, enfatizando no treino as qualidades mais importantes para a performance.

Um dos pontos fortes do estudo é a representatividade da amostra e a abordagem multidimensional utilizada na investigação dos fatores e indicadores do desempenho na GTR. Porém, cabe destacar que os resultados encontrados dizem respeito ao conhecimento e opinião de um determinado grupo de treinadores, gestores e árbitros. Além disso, outros fatores, tais como aspectos biomecânicos, maturacionais e genéticos não foram considerados. No fator socioambiental não foram considerados alguns indicadores sabidamente importantes para o desenvolvimento de jovens talentos, como por exemplo: idade de início, quantidade e qualidade da prática, qualidade dos treinadores, acesso a centros de treinamento, infraestrutura de treinamento, experiência prévia em outras modalidades, dentre outras. Tais aspectos devem ser investigados em estudos futuros.

\section{CONSIDERAÇÕES FINAIS}

Revelou-se a opinião dos experts quanto à importância dos fatores dos determinantes de desempenho na detecção e seleção de talentos na GTR. 
Concluindo, os principais fatores de desempenho de acordo com a opinião dos experts na GTR são os físico-motores e técnicos. Os indicadores de desempenho: massa muscular, velocidade, agilidade, flexibilidade, equilíbrio e força rápida de membros superiores têm diferenças significativas entre os aparelhos da modalidade. No TU os fatores antropométricos e fisicomotores tem maior grau de importância, no DMT o fator tático e no TRA foi considerado o fator técnico. Nosso estudo retrata os fatores de desempenho mais importantes para ser bem sucedido na Ginástica de Trampolim, com isso contribuímos com os determinantes indispensáveis para obter bons resultados nesta modalidade esportiva, assim como as características específicas para cada um dos seus três aparelhos.

\section{FinANCIAMENTO}

Agradecemos ao Programa de Bolsas de Iniciação Científica e Tecnológica Institucional da Fapemig (PIBIC/FAPEMIG/UFOP) - Edital 10/2017 e ao Programa de Iniciação à Pesquisa - Edital 24/2019-PIP-1S/UFOP-20-2019-6Meses, à Universidade Federal de Ouro Preto, Confederação Brasileira de Ginástica e Federação Mineira de Ginástica.

\section{NOTAS}

\section{CONFLITOS DE INTERESSE}

Os autores não têm conflitos de interesse, incluindo interesses financeiros específicos e relacionamentos e afiliações relevantes ao tema ou materiais discutidos no manuscrito.

\section{Autoria e CoAutoria}

Os autores declaram que participaram de forma significativa na construção e formação desde estudo, tendo, enquanto autores, responsabilidade pública pelo conteúdo deste, pois, contribuíram diretamente para o conteúdo intelectual deste trabalho e satisfazem as exigências de autoria.

Ana Luíza Ferreira - Concepção e desenvolvimento (desde a ideia para a investigação ou artigo, criou a hipótese); Coleta e tratamento dos dados (responsável pelos experimentos, pacientes, organização dos dados); Levantamento da literatura (participou da pesquisa bibliográfica e levantamento de artigos); Redação (responsável por escrever uma parte substantiva do manuscrito).

Paulo Carrara - Concepção e desenvolvimento (desde a ideia para a investigação ou artigo, criou a hipótese); Desenho metodológico (planejamento dos métodos para gerar os resultados); Levantamento da literatura (participou da pesquisa bibliográfica e levantamento de artigos); Revisão crítica (responsável pela revisão do conteúdo intelectual do manuscrito antes da apresentação final). 
Kerly Priscila Jesus de Oliveira - Desenho metodológico (planejamento dos métodos para gerar os resultados); Coleta e tratamento dos dados (responsável pelos experimentos, pacientes, organização dos dados); Levantamento da literatura (participou da pesquisa bibliográfica e levantamento de artigos); Revisão crítica (responsável pela revisão do conteúdo intelectual do manuscrito antes da apresentação final).

Newton Santos Vianna Júnior - Desenho metodológico (planejamento dos métodos para gerar os resultados); Levantamento da literatura (participou da pesquisa bibliográfica e levantamento de artigos); Revisão crítica (responsável pela revisão do conteúdo intelectual do manuscrito antes da apresentação final).

Francisco Zacaron Werneck - Concepção e desenvolvimento (desde a ideia para a investigação ou artigo, criou a hipótese); Desenho metodológico (planejamento dos métodos para gerar os resultados); Supervisão (responsável pela organização e execução do projeto e da escrita do manuscrito); Análise / interpretação (responsável pela análise estatística, avaliação e apresentação dos resultados); Revisão crítica (responsável pela revisão do conteúdo intelectual do manuscrito antes da apresentação final).

\section{REFERÊNCIAS}

AGUIAR, Caio M. Importância atribuída por treinadores aos determinantes do desempenho no atletismo. 2018. 32 f. Monografia (Graduação em Educação Física) Departamento de Educação Física, Universidade Federal de Ouro Preto, Ouro Preto, 2018.

ALBUQUERQUE, Patrícia Arruda de; FARINATTI, Paulo de Tarso Veras. Desenvolvimento e validação de um novo sistema de seleção de talentos para a ginástica olímpica feminina: a Bateria PDGO. Revista Brasileira de Medicina do Esporte, v. 13, n. 3, p. 157-164, 2007.

BACCIOTTI, Sarita; BAXTER-JONES, Adam; GAYA, Adroaldo; MAIA, José. Body physique and proportionality of Brazilian female artistic gymnasts. Journal of sports sciences, $v$. 36, n. 7, p. 749-756, 2018.

BACCIOTTI, Sarita; GAYA, Adroaldo; PEREIRA, Sara; GOMES, Thayse; BACCIOTTI, Franciscarlos; BAXTER-JONES, Adam; MAIA, José. Seleção em ginástica artística feminina no Brasil. Revista Brasileira de Ciências do Esporte, v. 41, n. 1, p. 51-58, 2019.

BÖHME, Maria Tereza Silveira. O treinamento a longo prazo de detecção, seleção e promoção de talentos esportivos. Revista brasileira de ciências do esporte. Revista brasileira de ciências do esporte, v. 21, n. 2, p. 4-10, 2000.

BOMPA, Tudor O. Periodização: teoria e metodologia do treinamento. São Paulo: Phorte, 2002.

BORTOLETO, Marco; CARRARA, Paulo; ROVERI, Murilo Guarniei. Trampoline gymnastics: the Brazilian participation at international championships-the olympic games still a dream. Science of Gymnastics Journal, v. 10, n. 3, p. 467-483, 2018.

BRESSEL, Eadric; YONKER, Joshua C.; KRAS, John; HEATH, Edward M. Comparison of static and dynamic balance in female collegiate soccer, basketball, and gymnastics athletes. Journal of athletic training, v. 42, n. 1, p. 42-46, 2007. 
CARR, Gerry. Mechanics of Sport: A Practitioner's Guide. Champaign, IL: Human Kinetics, 1997.

CBG, Confederação Brasileira de Ginástica. Regulamento Técnico Campeonatos Brasileiros - GTR - 2017. Aracaju: Confederação Brasileira de Ginástica, 2017.

CÔTÉ, Jean; SALMELA, John; TRUDEL, Pierre; BARIA, Abderrahim; RUSSEL, Storm. The Coaching Model: A grounded assessment of expert gymnastic coaches's knowledge Journal of Sport and Exercise Psychology, v. 17, n. 1, p. 1-17, 1995.

CRIPPS, Ashley J.; HOPPER, Luke S.; JOYCE, Christopher. Coaches' perceptions of longterm potential are biased by maturational variation. International Journal of Sports Science \& Coaching, v. 11, n. 4, p. 478-481, 2016.

DOUDA, Helen T.; TOUBEKIS, Argyris G.; AVLONITI, Alexandra A.; TOKMAKIDIS, Savvas P. Physiological and Anthropometric Determinants of Rhythmic Gymnastics Performance. International Journal of Sports Physiology and Performance, v. 3, n. 1, p. 41-54, 2008.

ESPOSITO, Paul W.; ESPOSITO, Lisa M. The reemergence of the trampoline as a recreational activity and competitive sport. Current Sports Medicine Reports, v. 8, n. 5, p. 273-277.

FARIA, Irvin E.; FARIA, Erik W. Relationship of the anthropometric and physical characteristics of male junior gymnasts to performance. Journal of Sports Medicine and Physical Fitness, v. 29, n. 4, p. 369-378, 1989.

FIG, Fédération Internationale de Gymnastique. 2017 - 2020 Code of Points: Trampoline Gymnastics. Lausanne: Fédération Internationale de Gymnastique, 2017.

FIG, Fédération Internationale de Gymnastique. Parkour: Presentation. Lausanne, 2019. Disponível em: www.gymnastics.sport/site/pages/disciplines/pres-pk.php. Acesso em: 16 out. 2020.

FIG, Fédération Internationale de Gymnastique. FIG Apparatus Norms. Lausanne: Fédération Internationale de Gymnastique, 2020.

FONTANA, Keila Elizabeth; SOARES, Edgard Keene von Koenig; SANTOS, Jônatas de Oliveira; MOLINA, Guilherme Eckhardt; RIEHL, Osmar. Antropometria e aptidão física de ginastas brasileiros de alto rendimento. Fitness \& performance journal, v. 10, n. 1, p. 1925, 2014.

GREENWOOD, Daniel; DAVIDS, Keith; RENSHAW, Ian. Experiential knowledge of expert coaches can help identify informational constraints on performance of dynamic interceptive actions. Journal of Sports Sciences, v. 32, n. 4, p. 328-335, 2014.

HIRATA, Ana Claudia de Souza; OLIVEIRA, Rodrigo Franco de. Protocolo de treinamento proprioceptivo para atletas de Ginástica Rítmica GR. Conscientiae saúde,São Paulo, v. 14, n. 4, p. 634-640, 2015.

KUNZE, Horst. World Age Group Competition Rules 2017 - 2020. Retrieved from FIG publications, Lausanne. Lausanne: FÉDÉRATION INTERNATIONALE DE GYMNASTIQUE: 14 p. 2019.

LANARO FILHO, Pedro; BÖHME, Maria Teresa Silveira. Detecção, seleção e promoção de talentos esportivos em ginástica rítmica desportiva: um estudo de revisão. Revista Paulista de Educação Física, São Paulo, v. 15, n. 2, p. 154-168, 2001. 
LARKIN, Paul; O'CONNOR, Donna. Talent identification and recruitment in youth soccer: Recruiter's perceptions of the key attributes for player recruitment. PLOS ONE, v. 12, n. 4, e0175716, 2017.

MALINA, Robert M.; BOUCHARD, Claude. Atividade física do atleta jovem: do crescimento à maturação. São Paulo: Editora Roca, 2002.

MASSUÇA, Luís; FRAGOSO, Isabel. Do talento ao alto rendimento: indicadores de acesso à excelência no handebol. Revista Brasileira de Educação Física e Esporte,São Paulo, v. 24, n. 4, p. 483-491, 2010.

MILLISTETD, Michel; MESQUITA, Isabel; SOUZA SOBRINHO, Antonio; CARRARA, Paulo; NASCIMENTO, Juarez V. Coaches representation about detection and selection of talents on the brazilian volleyball. International Journal of Sports Science, v. 3, n. 4, p. 157-162, 2013.

MOREIRA, Jorge; ARAÚJO, Carlos. Manual técnico e pedagógico de Trampolins. Porto: Porto Editora, 2004.

NUNOMURA, Myrian; CARRARA, Paulo; TSUKAMOTO, Mariana. Ginástica artística e especialização precoce: cedo demais para especializar, tarde demais para ser campeão! Revista brasileira de Educação Física e Esporte,São Paulo, v. 4, n. 3, p. 305-314, 2010.

NUNOMURA, Myrian; PIRES, Fernanda; CARRARA, Paulo. Análise do Treinamento na Ginástica Artística Brasileira. Revista Brasileira de Ciências do Esporte,Campinas, v. 31, n. 1 , p. 25-40, 2009.

OLIVEIRA, Kerly Priscila Jesus. Perfil multidimensional de jovens ginastas de trampolim brasileiros. 2018. 38 f. Monografia (Graduação em Educação Física) - Departamento de Educação Física, Universidade Federal de Ouro Preto, Ouro Preto, 2018.

PANKHURST, Anne; COLLINS, Dave. Talent Identification and Development: The Need for Coherence Between Research, System, and Process. Quest, v. 65, n. 1, p. 83-97, 2013.

SEREDYŃSKI, Antoni; POLAK, Ewa. Physical Fitness of Girls Practising Acrobatic and Trampoline Gymnastics Compared to that of Girls Practising other Sports in the Subcarpathian Province Team. Polish Journal of Sport and Tourism, v. 22, n. 3, p. 158$164,2015$.

SIAHKOUHIAN, Marefat; AALIZADEH, Bahman; ESMAEILZADE, S. Talent identification of elite Iranian male artistic and trampoline gymnasts. Middle-East Journal of Scientific Research, v. 16, n. 1, p. 51-54, 2013.

TSUKAMOTO, Mariana; NUNOMURA, Myrian; CARRARA, Paulo. Ginástica Artística. In: BÖHME, Maria Tereza Silveira (Ed.). Esporte infanto-juvenil: Treinamento a longo prazo e talento esportivo. São Paulo: Phorte, 2011. v. 1, cap. 18, p. 409-426.

UÇAN, Izzet. The Effect of Body Composition and Physical Fitness Factors on Performance Prediction of Elite Male Trampolines. Journal of Education and Training

Studies,Beaverton, v. 6, n. 4, p. 30-38, 2018.

WERNECK, Francisco Zacaron; FERREIRA, Renato Melo; COELHO, Emerson Filipino; SOBREIRA, Diana Izaías; PAULA, Hugo Leonardo Barros de; MIRANDA, Luciano; RIBAS, Paulo Roberto; FIGUEIREDO, António José Barata. Projeto atletas de ouro: validade e estabilidade do diagnóstico do potencial esportivo em escolares de um colégio militar. Revista de Educação Física, v. 86, n. 2, p. 139-141, 2017 a. 
WERNECK, Francisco Zacaron; COELHO, Emerson Filipino; SOARES, Everton Rocha; PAULA, Heber Eustáquio de; FERREIRA, Renato Melo. Projeto Atletas de Ouro: modelagem do potencial esportivo de jovens atletas de ginástica de trampolim: relatório técnico do estudo realizado no campeonato estadual e brasileiro por idades Ouro Preto MG/ julho 2017. Ouro Preto: UFOP, 2017b. p. 33. Disponível em:

http://www.repositorio.ufop.br/handle/123456789/11656. Acesso em: 16 out. 2020.

ZATSIORSKY, Vladimir M.; KRAEMER, William J. Science and Practice of Strength Training. 2nd ed. Champaign: Human Kinetics, 2006.

Artigo submetido ao sistema de similaridade Turnitin $®$.

A revista Conexões utiliza a Licença Internacional Creative Commons Atribuição 4.0, preservando assim, a integridade dos artigos em ambiente de acesso aberto.

A Revista Conexões é integrante do Portal de Periódicos Eletrônicos da Unicamp e associado/membro das seguintes instituições:

\section{NABEC \&}

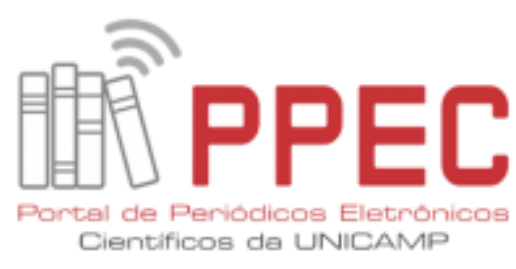

